\title{
Influence of seed position within the fruit on seedling quality and in vitro shoot tip production of jackfruit
}

\begin{abstract}
This study reports on the influence of seed position within the fruit and shoot development on the production of jackfruit in vitro. Seeds were extracted from the different sections in the fruit and shoot tips excised from the resultant seedlings. The tips were cultured in vitro on media supplemented with 6-benzylaminopurine (BAP) or thidiazuron (TDZ). The seeds from the middle and basal sections of the fruit were larger and produced larger seedlings than those from the top. Seeds from the basal position took 10 days to germinate and produced seedlings $11 \mathrm{~cm}$ tall with four or five leaves. Partially covered and fully open shoots from 6-8 week old seedlings performed best. The highest frequency of shoot induction (100\%) and multiple shoot production $(7.31 \pm 1.2)$ with a mean length of $3.6 \pm 0.13 \mathrm{~cm}$ was observed on Murashige and Skoog (MS) media supplemented with $3.0 \mathrm{mg} / \mathrm{L}$ BAP. Driver and Kuniyuki (DKW), and MS media were equally effective in shoot multiplication. More than $80 \%$ of the rooted plants survived culture, indicating that this technology could be used to conserve wild germplasm.
\end{abstract}

Keyword: Seed position; Seedling age; Shoot tips; Shoot multiplication; In vitro culture 